\title{
ON WEAKLY CHAINABLE INVERSE LIMITS WITH SIMPLICIAL BONDING MAPS
}

\author{
PIOTR MINC
}

\author{
(Communicated by James E. West)
}

\begin{abstract}
In this paper we prove that if an atriodic and weakly chainable continuum is the inverse limit of trees with simplicial bonding maps, then it is chainable.
\end{abstract}

By a graph we understand a one-dimensional polyhedron $G$ with finite and fixed collections of vertices $\mathscr{V}(G)$ and edges $\mathscr{E}(G)$. We will assume that every graph is a subset of three-dimensional Euclidean space and every edge is a straight linear closed segment between its vertices. Two vertices belonging to the same edge are called adjacent. A connected graph without a simple closed curve is called a tree. A tree in which every vertex belongs to at most two edges is called an arc. If $u$ and $v$ are two adjacent vertices of a graph, by $\langle u, v\rangle$ we will denote the edge between $u$ and $v$. Additionally, if $u$ and $v$ are two vertices of a tree, by $\langle u, v\rangle$ we will denote the arc between $u$ and $v$. A map $\varphi$ of a graph $G_{1}$ into a graph $G_{0}$ is simplicial provided that $\varphi\left(\mathscr{V}\left(G_{1}\right)\right) \subset \mathscr{V}\left(G_{0}\right)$, and if $u$ and $v$ are two adjacent vertices of $G_{1}$, then $\varphi \mid\langle u, v\rangle$ is a linear map either onto an edge or onto a single vertex.

All topological spaces considered in this paper are metric. A continuum is a connected and compact space. A continuum is tree-like (chainable) provided that it can be expressed as the inverse limit of a sequence of trees (arcs) with continuous (not necessarily simplicial) bonding maps. A continuum is weakly chainable if it is a continuous image of a chainable continuum (see [3, 2]). We will say that three continua $A, B$, and $C$ form a triod if none of them is contained in the union of the remaining two and $\varnothing \neq A \cap B \cap C=A \cap B=$ $A \cap C=B \cap C$. A continuum is atriodic if it does not contain a triod.

In 1981 Mohler asked whether every weakly chainable atriodic tree-like continuum $X$ is chainable (see [16, Problem 171;6, Problem 16]). This problem is closely related to the question of whether span (see $[4,5])$ zero implies chainability (see [1, Problem \#8]). Both problems were extensively studied by Oversteegen and Tymchatyn in [11-15] and by Oversteegen in [10]. Among other results Oversteegen and Tymchatyn proved in $[11,14]$ that any tree-like

Received by the editors January 26, 1991.

1991 Mathematics Subject Classification. Primary 54F15.

Key words and phrases. Continua, chainable, weakly chainable, atriodic, simplicial maps, graphs, trees. 
homogeneous plane continuum is weakly chainable. Thus a positive answer to Mohler's question would complete the characterization of homogeneous plane continua. Oversteegen gave a partial positive answer to the question, assuming additionally that the surjective semispan of $X$ is zero and that $X$ is the continuous image of a chainable continuum $C$ under an induced map, i.e., a map which is induced by a commutative diagram of maps between inverse systems defining $X$ and $C$ [10, Theorem 5.1]. In this paper we give another partial answer to Mohler's question, assuming that $X$ is the inverse limit of a system of trees with simplicial bonding maps (see Theorem 2). Note that in this case it follows that $X$ is a continuous image of a chainable continuum under an induced map (Proposition 1). So the real difference between Oversteegen's theorem and Theorem 2 is a trade of the assumption that the surjective semispan of $X$ is zero for simplicial bonding maps in the inverse system defining $X$.

In [7] the author introduced an operation $d$ assigning to each simplicial map $\varphi$ between graphs a simplicial map $d[\varphi]$ between another pair of graphs. Simplicial maps that can be factored through an arc were characterized in terms of the operation [7, Theorems 2.12, 2.13, and 2.18]. The proof of Theorem 2 presented here is based on those characterizations. Another application of the technique was shown in [7] to prove that surjective span zero is equivalent to chainability for inverse limits of trees with simplicial bonding maps [7, Theorem 3.3]. The last result is also very closely related to Oversteegen's Theorem 5.1 from [10]. (Oversteegen announced a similar result, with surjective semispan replaced by surjective span, at the Prague Topological Symposium, Czechoslovakia, 1986. The proof can be inferred from [10, Theorem 5.1].) Even though the respective proofs of [10, Theorem 5.1; 7, Theorem 3.3] and Theorem 2 from this paper are different, the combinatorial methods underlining them have something in common. In his proof, Oversteegen considers tree-words (trees with vertices labeled by letters) and uses the methods developed in $[8,9]$ to reduce tree-words to chain-words. Note that a tree-word can be associated with a simplicial map which takes each vertex $v$ of the tree-word to the letter labeling $v$. One can see that a tree-word can be reduced to a chain-word if the simplicial map associated with it can be factored through an arc. It is conceivable that this similarity can be used in combining the arguments to prove that the image of a chainable continuum under an induced map is chainable.

Proposition 1. Let $\left\{G_{j}, \varphi_{j}^{i}\right\}_{j=0}^{\infty}$ be an inverse system of graphs with simplicial and surjective bonding maps $\varphi_{j}^{i}: G_{i} \rightarrow G_{j}$. Let $X$ denote the inverse limit (in the topological sense) of $\left\{G_{j}, \varphi_{j}^{i}\right\}_{j=0}^{\infty}$. Suppose that $X$ is weakly chainable. Then there is an inverse system $\left\{A_{j}, \alpha_{j}^{i}\right\}_{j=0}^{\infty}$ of simplicial arcs with simplicial bonding maps and there is a sequence of simplicial and surjective maps $\sigma_{j}: A_{j} \rightarrow G_{j}$, $j=0,1, \ldots$, such that $\sigma_{j} \circ \alpha_{j}^{i}=\varphi_{j}^{i} \circ \sigma_{i}$ for every $i \geq j$.

Proof. Let $p_{j}$ denote the projection of $X$ onto $G_{j}$. We will assume that each directed edge $\langle v, w\rangle$ of $G_{j}$ is uniformly parametrized by the interval $[0,1]$. Let $Y_{j}^{\mu}(v, w)$ denote the open segment parametrized by $\left(\frac{\mu}{4}, \frac{\mu+1}{4}\right)$ for $\mu=$ $0,1,2,3$. Observe that $Y_{j}^{\mu}(v, w)=Y_{j}^{3-\mu}(w, v)$. Note that $\varphi_{j}^{i}\left(Y_{i}^{\mu}(v, w)\right)=$ $Y_{j}^{\mu}\left(\varphi_{j}^{i}(v), \varphi_{j}^{i}(w)\right)$ for $i>j$ and any pair $v, w$ of adjacent vertices of $G_{i}$. For any vertex $v \in \mathscr{V}\left(G_{j}\right)$, let $Y_{j}(v)$ be the union of $\{v\}$ and all segments $Y_{j}^{0}(v, w)$ with $w$ adjacent to $v$. 
Let $f$ be a continuous map of a chainable continuum $P$ onto $X$. We will say that $\mathscr{U}=\left\{U_{0}, U_{1}, \ldots, U_{m}\right\}$ is a taut chain covering $P$ provided that the sets $U_{0}, U_{1}, \ldots, U_{m}$ are open in $P$, their union is $P$, and $\operatorname{cl}\left(U_{k}\right) \cap \operatorname{cl}\left(U_{n}\right) \neq \varnothing$ if and only if $|k-n| \leq 1$ (where $\operatorname{cl}(U)$ denotes the closure of $U$ ). Since $P$ is chainable there are taut chains covering $P$ with arbitrarily small mesh.

There is a taut chain $\mathscr{U}_{0}=\left\{U_{0}^{0}, U_{1}^{0}, \ldots, U_{m(0)}^{0}\right\}$ covering $P$ such that for each $k=0,1, \ldots, m(0)$ we have

(i) either $p_{0}\left(f\left(U_{k}^{0}\right)\right)$ is contained in an open edge of $G_{0}$ or there is a vertex $v \in \mathscr{V}\left(G_{0}\right)$ such that $p_{0}\left(f\left(U_{k}^{0}\right)\right) \subset Y_{0}(v)$, and

(ii) $)_{0}$ if $v$ and $w$ are two adjacent vertices of $G_{0}$ and $p_{0}\left(f\left(U_{k}^{0}\right)\right)$ contains the midpoint of the segment $Y_{0}^{1}(v, w)$, then $p_{0}\left(f\left(U_{k}^{0}\right)\right) \subset Y_{0}^{1}(v, w)$.

Note that to satisfy the above two conditions it is enough to choose any taut chain $\mathscr{U}_{0}$ with sufficiently small mesh.

If $\mathscr{B}=\left\{B_{0}, B_{1}, \ldots, B_{k}\right\}$ and $\mathscr{C}=\left\{C_{0}, C_{1}, \ldots, C_{n}\right\}$ are two chains covering $P$, we say that $\mathscr{C}$ follows the pattern of $\mathscr{B}$ provided that $\mathscr{C}$ refines $\mathscr{B}$, and for all integers $i, i^{\prime}, i^{\prime \prime}, m^{\prime}$, and $m^{\prime \prime}$ such that $0 \leq i^{\prime}<i<i^{\prime \prime} \leq k$, $0 \leq m^{\prime} \leq n, 0 \leq m^{\prime \prime} \leq n, B_{i^{\prime}} \cap C_{m^{\prime}} \neq \varnothing$, and $B_{i^{\prime \prime}} \cap C_{m^{\prime \prime}} \neq \varnothing$, there is an integer $m$ between $m^{\prime}$ and $m^{\prime \prime}$ such that $C_{m} \subset B_{i}$ and $C_{m} \cap B_{j}=\varnothing$ for $j \neq i$. Observe that if $\mathscr{C}$ refines $\mathscr{B}$ and it takes at least three links of $\mathscr{C}$ to stretch between nonadjacent links of $\mathscr{B}$, then $\mathscr{C}$ follows the pattern of $\mathscr{B}$. It follows that for each taut chain $\mathscr{B}$ there is a positive number $\varepsilon$ such that if $\mathscr{C}$ is an arbitrary chain covering $P$ and the mesh of $\mathscr{C}$ is less than $\varepsilon$, then $\mathscr{C}$ follows the pattern of $\mathscr{B}$.

By choosing one by one taut chains with sufficiently small mesh, we construct a sequence of taut chains $\mathscr{U}_{0}, \mathscr{U}_{1}, \ldots, \mathscr{U}_{j}=\left\{U_{0}^{j}, U_{1}^{j}, \ldots, U_{m(j)}^{j}\right\}, \ldots$, each covering $P$ such that $\mathscr{U}_{j}$ follows the pattern of $\mathscr{U}_{j-1}$, and for each $k=0,1, \ldots, m(j)$ we have

(i) $)_{j}$ either $p_{j}\left(f\left(U_{k}^{j}\right)\right)$ is contained in an open edge of $G_{j}$ or there is a vertex $v \in \mathscr{V}\left(G_{j}\right)$ such that $p_{j}\left(f\left(U_{k}^{j}\right)\right) \subset Y_{j}(v)$.

It follows by induction from (ii) $)_{0}$ that

(ii) $j$ if $v$ and $w$ are two adjacent vertices of $G_{j}$ and $p_{j}\left(f\left(U_{k}^{j}\right)\right)$ contains the midpoint of the segment $Y_{j}^{1}(v, w)$, then $p_{j}\left(f\left(U_{k}^{j}\right)\right) \subset Y_{j}^{1}(v, w)$.

We will define recursively a sequence $Z_{0}, Z_{1}, \ldots$ of subcollections of $\mathscr{U}_{0}$, $\mathscr{U}_{1}, \ldots$, respectively. Let $Z_{0}$ be the set of the elements $U_{k}^{0}$ of $\mathscr{U}_{0}$ such that either $p_{0}\left(f\left(U_{k}^{0}\right)\right) \subset Y_{0}(v)$ for some vertex $v \in \mathscr{V}\left(G_{0}\right)$, or $p_{0}\left(f\left(U_{k}^{0}\right)\right) \subset Y_{0}^{1}(v, w)$ for some pair of adjacent vertices $v, w \in \mathscr{V}\left(G_{0}\right)$. Let $Z_{j}$ be the set of the elements $U_{k}^{j}$ of $\mathscr{U}_{j}$ such that $U_{k}^{j}$ is contained in some element of $Z_{j-1}$. It follows by induction that if $p_{0}\left(f\left(U_{k}^{j}\right)\right)$ contains either a vertex of $G_{j}$ or the midpoint of the segment $Y_{j}^{1}(v, w)$ for a pair of adjacent vertices $v$ and $w$ of $G_{j}$, then $U_{k}^{j} \in Z_{j}$.

Let $A_{j}$ denote the graph with $Z_{j}$ as its set of vertices such that two vertices $z_{1}, z_{2} \in Z_{j}$ are adjacent if the subchain of $\mathscr{U}_{j}$ between $z_{1}$ and $z_{2}$ does not contain other elements of $Z_{j}$. Clearly, $A_{j}$ is an arc. Define $\alpha_{j-1}^{j}: A_{j} \rightarrow A_{j-1}$ by setting $\alpha_{j-1}^{j}(z)$ to be any element of $Z_{j-1}$ containing $z$, for $z \in Z_{j}$. 
Note that this definition is not unique. We will prove that $\alpha_{j-1}^{j}$ is simplicial (regardless of the choice of $\alpha_{j-1}^{j}(z)$ ).

Let $z_{1}$ and $z_{2}$ be any two adjacent vertices of $A_{j}$. Suppose that $\alpha_{j-1}^{j}\left(z_{1}\right)$ and $\alpha_{j-1}^{j}\left(z_{2}\right)$ are two different vertices which are not adjacent in $A_{j-1}$. Then the subchain of $\mathscr{U}_{j-1}$ between $\alpha_{j-1}^{j}\left(z_{1}\right)$ and $\alpha_{j-1}^{j}\left(z_{2}\right)$ contains an element $u \in Z_{j-1}$ such that $\alpha_{j-1}^{j}\left(z_{1}\right) \neq u \neq \alpha_{j-1}^{j}\left(z_{2}\right)$. Since $\mathscr{U}_{j}$ follows the pattern of $\mathscr{U}_{j-1}$, the subchain of $\mathscr{U}_{j}$ between $z_{1}$ and $z_{2}$ contains an element $z$, such that $z \subseteq u$ and $z$ does not intersect $\alpha_{j-1}^{j}\left(z_{1}\right) \cup \alpha_{j-1}^{j}\left(z_{2}\right)$. It follows that $z \in Z_{j}$ and $z_{1} \neq z \neq z_{2}$. Thus, $z_{1}$ and $z_{2}$ are not adjacent. Consequently, $\alpha_{j-1}^{j}$ is simplicial.

It follows by induction from the definition of $Z_{0}$ and the condition $(i)_{j}$ that if $z \in Z_{j}$, then either there is a vertex $s \in \mathscr{V}\left(G_{j}\right)$ such that $p_{j}(f(z)) \subset$ $Y_{j}(s)$, or there are two adjacent vertices $v$ and $w$ of $G_{j}$ such that $p_{j}(f(z)) \subset$ $Y_{j}^{1}(v, w)$. If $p_{j}(f(z)) \subset Y_{j}(s)$, then define $\sigma_{j}(z)=s$. If $p_{j}(f(z)) \subset Y_{j}^{1}(v, w)$, then define $\sigma_{j}(z)=w$. Clearly, $\sigma_{j-1} \circ \alpha_{j-1}^{j}=\varphi_{j-1}^{j} \circ \sigma_{j}$. We will prove that $\sigma$ is a simplicial map. Let $z=U_{k}^{j}$ and $z^{\prime}=U_{k^{\prime}}^{j}$ be two adjacent vertices of $A_{j}$ and suppose that $\sigma_{j}(z)$ and $\sigma_{j}\left(z^{\prime}\right)$ are two different and not adjacent vertices of $G_{j}$. There is a component $C$ of the union $U_{k}^{j} \cup U_{k+1}^{j} \cup \cdots \cup U_{k^{\prime}}^{j}$ such that $C \cap U_{k}^{j} \neq \varnothing \neq C \cap U_{k^{\prime}}^{j}$. Now, we will consider two cases.

Case I. There is a vertex $s \in \mathscr{V}\left(G_{j}\right)$ such that $p_{j}(f(z)) \subset Y_{j}(s)$.

Case II. Neither $p_{j}(f(z))$ nor $p_{j}\left(f\left(z^{\prime}\right)\right)$ is contained in $Y_{j}(s)$ for any vertex $s \in \mathscr{V}\left(G_{j}\right)$.

In the first case there is a vertex $t \in \mathscr{V}\left(G_{j}\right)$ adjacent to $s$ such that the midpoint of the segment $Y_{j}^{1}(s, t)$ belongs to $p_{j}(f(C))$, and thus, there is an integer $i$ such that $k<i<k^{\prime}$ and $U_{i}^{j} \in Z_{j}$. This means that $z$ and $z^{\prime}$ are not adjacent. In Case II, let $v$ and $w$ be two adjacent vertices of $G_{j}$ such that $p_{j}(f(z)) \subset$ $Y_{j}^{1}(v, w)$. If $p_{j}\left(f\left(z^{\prime}\right)\right) \subset\langle v, w\rangle$, then $\sigma_{j}(z)$ and $\sigma_{j}\left(z^{\prime}\right)$ are adjacent. So we can assume that $p_{j}(f(C))$ contains at least one of the vertices $v$ and $w$. There is an integer $i$ such that $k<i<k^{\prime}$ and $p_{j}\left(f\left(U_{i}^{j}\right)\right)$ contains either $v$ or $w$, and we again have the result that $U_{i}^{j} \in Z_{j}$. Hence, $\sigma_{j}$ is a simplicial map.

To complete the proof it remains to show that $\sigma_{j}$ is surjective. Let $v$ and $w$ be a pair of adjacent vertices of $G_{j}$. Let $m_{1}$ denote the midpoint of the segment $Y_{j}^{1}(v, w)$ and let $m_{2}$ denote the midpoint of the segment $Y_{j}^{2}(v, w)$. There is a point $x \in P$ such that $p_{j}(f(x))=m_{1}$. There is an integer $i$ such that $x \in U_{i}^{j}$. Observe that $U_{i}^{j} \in Z_{j}$ and $\sigma_{j}\left(U_{i}^{j}\right)=w$. Let $S$ denote the subsegment of $\langle v, w\rangle$ between $v$ and $m_{2}$, and let $C$ be the component of $x$ in $\left(p_{j} \circ f\right)^{-1}(S)$. There is a point $y \in C$ such that either $p_{j}(f(y))=m_{1}$ or $p_{j}(f(y))=v$. There is an integer $k$ such that $y \in U_{k}^{j}$. Note that $U_{k}^{j} \in Z_{j}$ and $\sigma_{j}\left(U_{k}^{j}\right)=v$. It follows from (i) ${ }_{j}$ that every element of $Z_{j}$ in the chain between $U_{i}^{j}$ and $U_{k}^{j}$ is mapped by $\sigma_{j}$ onto either $v$ or $w$. Thus the chain between $U_{i}^{j}$ and $U_{k}^{j}$ contains two elements $z$ and $z^{\prime}$ which are adjacent in $A_{j}$ and such that $\sigma_{j}\left(\left\langle z, z^{\prime}\right\rangle\right)=\langle v, w\rangle$. 
Proposition 2. Let $\left\{G_{j}, \varphi_{j}^{i}\right\}_{j=0}^{\infty}$ be an inverse system of graphs with simplicial bonding maps $\varphi_{j}^{i}: G_{i} \rightarrow G_{j}$. Let $S_{j}$ be a collection of subgraphs of $G_{j}$ for each $j=0,1, \ldots$. Suppose that if $i>j$ and $g \in S_{i}$, then $\varphi_{j}^{i}(g) \in S_{j}$. Then there is a sequence $g_{0}, g_{1}, g_{2}, \ldots$, such that $g_{j} \in S_{j}$ and $\varphi_{j}^{i}\left(g_{i}\right)=g_{j}$ for all integers $j$ and $i$ such that $i>j \geq 0$.

We need to recall the following definitions from [7].

Definition. For a graph $G_{0}$, let $D\left(G_{0}\right)$ denote the graph such that

(1) the set of vertices of $D\left(G_{0}\right)$ consists of edges of $G_{0}$, and

(2) two vertices of $D\left(G_{0}\right)$ are adjacent if and only if they intersect (as edges of $\left.G_{0}\right)$.

Let $\varphi: G_{1} \rightarrow G_{0}$ be a simplicial map between graphs. For every (closed) edge $e \in \mathscr{E}\left(G_{0}\right)$, let $\mathscr{K}(e)$ denote the set of components of $\varphi^{-1}(e)$ which are mapped by $\varphi$ onto $e$. Denote by $\mathscr{K}(\varphi)$ the union of all $\mathscr{K}(e)$. Let $D\left(\varphi, G_{1}\right)$ be the graph such that

(iii) the vertices of $D\left(\varphi, G_{1}\right)$ are elements of $\mathscr{K}(\varphi)$, and

(iv) two vertices of $D\left(\varphi, G_{1}\right)$ are adjacent if and only if they intersect (as subgraphs of $\left.G_{1}\right)$.

Let $d[\varphi]: D\left(\varphi, G_{1}\right) \rightarrow D\left(G_{0}\right)$ be the map defined by the formula $d[\varphi](v)=$ $\varphi(v)$ for every vertex $v$ of $D\left(\varphi, G_{1}\right)$.

Every vertex $v \in \mathscr{V}\left(D\left(\varphi, G_{1}\right)\right)$ is also a subgraph of $G_{1}$. To avoid confusion we will denote this subgraph by $v^{*}$.

The map $d[d[\varphi]]$ will be denoted by $d^{2}[\varphi]$, and recursively $d\left[d^{n-1}[\varphi]\right]$ will be denoted by $d^{n}[\varphi]$. The domain of $d^{n}[\varphi]$ will be denoted by $D^{n}\left(\varphi, G_{1}\right)$ and the range by $D^{n}\left(G_{0}\right)$.

Let $\psi$ be simplicial maps of a graph $G_{2}$ into $G_{1}$. Let $d[\varphi \psi]$ : $D\left(\varphi \circ \psi, G_{2}\right) \rightarrow D\left(\varphi, G_{1}\right)$ be the map such that for every vertex $v$ of $D\left(\varphi \circ \psi, G_{2}\right), d[\varphi, \psi](v)$ is the vertex of $D\left(\psi, G_{1}\right)$ containing $\psi\left(v^{*}\right)$. Let $d^{n}[\varphi, \psi]: D^{n}\left(\varphi \circ \psi, G_{2}\right) \rightarrow D^{n}\left(\varphi, G_{1}\right)$ denote the map defined by the formula $d^{n}[\varphi, \psi]=d\left[d^{n-1}[\varphi], d^{n-1}[\varphi, \psi]\right]$.

Proposition 3. Let $\varphi: T \rightarrow G$ be a simplicial map of a tree $T$ into a graph $G$. Suppose that $D(\varphi, T)$ contains a simple triangle $a, b, c$. (A simple triangle is a graph with three vertices and three edges.) Then the edges $\varphi\left(a^{*}\right), \varphi\left(b^{*}\right)$, and $\varphi\left(c^{*}\right)$ have a common vertex $v$. Moreover, there is a connected subgraph $Y$ of $T$ and there are three edges $A, B, C \in \mathscr{E}(T)$ such that $A \cap Y \neq \varnothing, B \cap Y \neq \varnothing$, $C \cap Y \neq \varnothing, \varphi(Y)=v, \varphi(A)=\varphi\left(a^{*}\right), \varphi(B)=\varphi\left(b^{*}\right)$, and $\varphi(C)=\varphi\left(c^{*}\right)$.

Proof. Note that each of the sets $a^{*}, b^{*}$, and $c^{*}$ is a tree. Since $a, b$, and $c$ are vertices of a simple triangle, the sets $a^{*} \cap b^{*}, a^{*} \cap c^{*}$, and $b^{*} \cap c^{*}$ are not empty. It follows that $b^{*} \cup c^{*}$ is a tree and the intersection of $a^{*}$ and $b^{*} \cup c^{*}$ is connected. Since $a^{*} \cap\left(b^{*} \cup c^{*}\right)=\left(a^{*} \cap b^{*}\right) \cup\left(a^{*} \cap c^{*}\right)$, the sets $a^{*} \cap b^{*}$ and $a^{*} \cap c^{*}$ must intersect. So $a^{*}, b^{*}$, and $c^{*}$ contain a common vertex $u$. Let $v=\varphi(u)$ and let $Y$ be the component of $\varphi^{-1}(v)$ containing $u$. Let $A(B$ and $C)$ be an edge of $a^{*}\left(b^{*}\right.$ and $c^{*}$, respectively) intersecting $Y$, but not contained in $Y$. Observe that $\varphi(A)=\varphi\left(a^{*}\right), \varphi(B)=\varphi\left(b^{*}\right)$, and $\varphi(C)=\varphi\left(c^{*}\right)$.

Proposition 4. Let $\varphi: T_{1} \rightarrow G$ be a simplicial map of a tree $T_{1}$ into a graph $G$ such that $D\left(\varphi, T_{1}\right)$ is a tree and let $\psi: T_{2} \rightarrow T_{1}$ be a simplicial map of a tree $T_{2}$ into $T_{1}$. Suppose that there is a connected subgraph $\widetilde{Y}$ of $D\left(\varphi \circ \psi, T_{2}\right)$ and there 
are three edges $\tilde{A}, \widetilde{B}, \widetilde{C} \in \mathscr{E}\left(D\left(\varphi \circ \psi, T_{2}\right)\right)$ such that $\tilde{A} \cap \tilde{Y} \neq \varnothing, \widetilde{B} \cap \tilde{Y} \neq \varnothing$, $\widetilde{C} \cap \widetilde{Y} \neq \varnothing$, and none of the sets $d[\varphi, \psi](\widetilde{A}), d[\varphi, \psi](\widetilde{B})$, and $d[\varphi, \psi](\widetilde{C})$ is contained in the union of the two remaining sets and $d[\varphi, \psi](\widetilde{Y})$. Then there is a connected subtree $Y$ of $T_{2}$ and there are three edges $A, B, C \in \mathscr{E}\left(T_{2}\right)$ such that $A \cap Y \neq \varnothing, B \cap Y \neq \varnothing, C \cap Y \neq \varnothing$, and none of the sets $\psi(A), \psi(B)$, and $\psi(C)$ is contained in the union of the two remaining sets and $\psi(Y)$. In particular, it follows that $\psi(A), \psi(B)$, and $\psi(C)$ are edges of $T_{1}$.

Proof. Each vertex $v$ of $D\left(\varphi \circ \psi, T_{2}\right)$ represents a subtree $v^{*}$ of $T_{2}$. Let $Y$ be the union of the subtrees of $T_{2}$ represented by vertices belonging to $\widetilde{Y}$. Let $a, b$, and $c$ denote the vertices of $\widetilde{A}, \widetilde{B}$, and $\widetilde{C}$, respectively, which do not belong to $\widetilde{Y}$. We will prove the following claim.

Claim (a). If $A$ is an edge contained in $a^{*}$ such that $\varphi \circ \psi(A)=\varphi \circ \psi\left(a^{*}\right)$, then $\psi(A)$ is not contained in $\psi\left(Y \cup b^{*} \cup c^{*}\right)$.

Suppose that the claim is false. Then there is a vertex $y$ of $\tilde{Y} \cup \widetilde{B} \cup \widetilde{C}$ such that $\psi(A)$ is contained in $\psi\left(y^{*}\right)$. It follows that $d[\varphi, \psi](a)=d[\varphi, \psi](y)$. Let $\tilde{a}$ denote the vertex of $\widetilde{A}$ different than $a$. Clearly, $\tilde{a}$ belongs to $\widetilde{Y}$. So both ends of $d[\varphi, \psi](\widetilde{A})$ belong to $d[\varphi, \psi](\widetilde{Y} \cup \widetilde{B} \cup \widetilde{C})$. Since $D\left(\varphi, T_{1}\right)$ is a tree, $d[\varphi, \psi](\widetilde{A})$ is contained in $d[\varphi, \psi](\widetilde{Y} \cup \widetilde{B} \cup \widetilde{C})$. This contradiction completes the proof of the claim.

In particular, it follows from the claim that $a^{*}$ is not contained in $Y$. Let $A$ be an edge of $a^{*}$ such that $A$ intersects $Y$ but is not contained in $Y$. Let $y$ be an element of $Y$ such that $y^{*}$ intersects $A$. Suppose $\varphi \circ \psi(A)$ is degenerate. Then $\varphi \circ \psi(A)$ belongs to $\varphi \circ \psi\left(y^{*}\right)$ and $A$ is contained in $Y^{*}$, because $y^{*}$ is the component of $\varphi^{-1}\left(\varphi \circ \psi\left(y^{*}\right)\right)$ containing the intersection of $A$ and $y^{*}$. Hence $\varphi \circ \psi(A)=\varphi \circ \psi\left(a^{*}\right)$ and the claim applies.

By a similar argument we can prove the corresponding claims for $b$ and $c$, and then choose $B$ and $C$.

Definition. Let $\left\{G_{j}, \varphi_{j}^{i}\right\}_{j=0}^{\infty}$ be an inverse system of graphs with simplicial bonding maps $\varphi_{j}^{i}: G_{i} \rightarrow G_{j}$. Suppose that each $G_{j}$ contains a connected subgraph $Y_{j}$ and three edges $A_{j}, B_{j}$, and $C_{j}$ intersecting it such that $\varphi_{j}^{i}\left(Y_{i}\right)=Y_{j}$, $\varphi_{j}^{i}\left(A_{i}\right)=A_{j}, \varphi_{j}^{i}\left(B_{i}\right)=B_{j}$, and $\varphi_{j}^{i}\left(C_{i}\right)=C_{j}$ for each $i>j \geq 0$. The system $\left\{Y_{j}, A_{j}, B_{j}, C_{j}\right\}_{j=0}^{\infty}$ is called a short triod if there is an integer $k$ such that none of the edges $A_{k}, B_{k}$, and $C_{k}$ is contained in the union of the remaining two and $Y_{k}$.

Proposition 5. Let $\left\{G_{j}, \varphi_{j}^{i}\right\}_{j=0}^{\infty}$ be an inverse system of graphs with simplicial bonding maps $\varphi_{j}^{i}: G_{i} \rightarrow G_{j}$. Suppose that there is an integer $k$ such that $G_{j}$ and $D\left(\varphi_{0}^{j}, G_{j}\right)$ are trees for each $j \geq k$. Suppose that the system $\left\{D\left(\varphi_{0}^{j}, G_{j}\right), d\left[\varphi_{0}^{j}, \varphi_{j}^{i}\right]\right\}_{j=0}^{\infty}$ contains a short triod $\left\{\tilde{Y}_{j}, \widetilde{A}_{j}, \widetilde{B}_{j}, \tilde{C}_{j}\right\}_{j=0}^{\infty}$. Then the system $\left\{G_{j}, \varphi_{j}^{i}\right\}_{j=0}^{\infty}$ also contains a short triod.

Proof. Without loss of generality we can assume that none of the edges $\widetilde{A}_{k}, \widetilde{B}_{k}$, and $\widetilde{\widetilde{C}}_{k}$ is contained in the union of the remaining two and $\widetilde{Y}_{k}$. By Proposition 4 , if $j>k$, then $G_{j}$ contains a subtree $Y_{j}$ and three edges $A_{j}, B_{j}$, and $C_{j}$ intersecting it such that $\varphi_{k}^{j}\left(A_{j}\right), \varphi_{k}^{j}\left(B_{j}\right)$, and $\varphi_{k}^{j}\left(C_{j}\right)$ are edges of $G_{k}$ and none of them is contained in the union of the remaining two and $\varphi_{k}^{j}\left(Y_{j}\right)$. 
Using Proposition 2, we can make $\varphi_{j}^{i}\left(Y_{i}\right)=Y_{j}$ for each $i>j>k$. Applying Proposition 2 three more times, we can make $\varphi_{j}^{i}\left(A_{i}\right)=A_{j}, \varphi_{j}^{i}\left(B_{i}\right)=B_{j}$, and $\varphi_{j}^{i}\left(C_{i}\right)=C_{j}$ for each $i>j>k$.

Theorem 1. Let $\left\{T_{j}, \varphi_{j}^{i}\right\}_{j=0}^{\infty}$ be an inverse system of trees with simplicial bonding maps $\varphi_{j}^{i}: T_{i} \rightarrow T_{j}$. Suppose that the inverse limit (in the topological sense) of $\left\{T_{j}, \varphi_{j}^{i}\right\}_{j=0}^{\infty}$ is atriodic. Then for every positive integer $n$ there is a positive integer $k$ such that $D^{n}\left(\varphi_{0}^{j}, T_{j}\right)$ is a tree for each $j \geq k$.

Proof. Suppose that the theorem is false. Let $n$ be the least positive integer such that $D^{n}\left(\varphi_{0}^{j}, T_{j}\right)$ is not a tree for infinitely many positive integers $j$. By Propositions 2.15 and 2.16 in [7], if the graph $D^{n}\left(\varphi_{0}^{j}, T_{j}\right)$ is not a tree, it contains a simple triangle. By [7, 1.14], $d^{n}\left[\varphi_{0}^{j}\right]$ maps every simple triangle in $D^{n}\left(\varphi_{0}^{i}, T_{j}\right)$ onto a simple triangle $D^{n}\left(T_{0}\right)$. Since $d^{n}\left[\varphi_{0}^{j}\right]=d^{n}\left[\varphi_{0}^{j-1}\right] \circ d^{n}\left[\varphi_{0}^{j-1}, \varphi_{j-1}^{j}\right]$, it follows that $d^{n}\left[\varphi_{0}^{j-1}, \varphi_{j-1}^{j}\right]$ maps every simple triangle in $D^{n}\left(\varphi_{0}^{j}, T_{j}\right)$ onto a simple triangle $D^{n}\left(\varphi_{0}^{j-1}, T_{j-1}\right)$. In particular, $D^{n}\left(\varphi_{0}^{j}, T_{j}\right)$ contains a simple triangle for each positive integer $j$. By Proposition 2 , in each $D^{n}\left(\varphi_{0}^{j}, T_{j}\right)$ we can choose a simple triangle with vertices $a_{j}, b_{j}$, and $c_{j}$ such that $d^{n}\left[\varphi_{0}^{j-1}, \varphi_{j-1}^{j}\right]\left(a_{j}\right)=$ $a_{j-1}, d^{n}\left[\varphi_{0}^{j-1}, \varphi_{j-1}^{j}\right]\left(b_{j}\right)=b_{j-1}$, and $d^{n}\left[\varphi_{0}^{j-1}, \varphi_{j-1}^{j}\right]\left(c_{j}\right)=c_{j-1}$ for each positive integer $j$.

By the choice of $n$, there is a positive integer $k$ such that $D^{n-1}\left(\varphi_{0}^{j}, T_{j}\right)$ is a tree for each $j \geq k$. Let $e_{a}, e_{b}$, and $e_{c}$ denote the edges $d^{n-1}\left[\varphi_{0}^{k}\right]\left(a_{k}^{*}\right)$, $d^{n-1}\left[\varphi_{0}^{k}\right]\left(b_{k}^{*}\right)$, and $d^{n-1}\left[\varphi_{0}^{k}\right]\left(c_{k}^{*}\right)$, respectively. Clearly, $e_{a}, e_{b}$, and $e_{c}$ are three different edges of $D^{n-1}\left(T_{0}\right)$. By Proposition $3, e_{a}, e_{b}$, and $e_{c}$ have a common vertex $v$. Again by Proposition 3, for each integer $j \geq k$, there is a subgraph $\widetilde{Y}_{j}$ of $D^{n-1}\left(\varphi_{0}^{j}, T_{j}\right)$ and there are three edges $\widetilde{A}_{j}, \widetilde{B}_{j}, \widetilde{C}_{j} \in \mathscr{E}\left(D^{n-1}\left(\varphi_{0}^{j}, T_{j}\right)\right)$ such that $\tilde{A}_{j} \cap \widetilde{Y}_{j} \neq \varnothing, \widetilde{B}_{j} \cap \tilde{Y}_{j} \neq \varnothing, \widetilde{C}_{j} \cap \widetilde{Y}_{j} \neq \varnothing, \varphi\left(\widetilde{Y}_{j}\right)=v, \varphi\left(\tilde{A}_{j}\right)=$ $e_{a}, \varphi\left(\widetilde{B}_{j}\right)=e_{b}$, and $\varphi\left(\widetilde{C}_{j}\right)=e_{c}$. By Proposition 2, we can assume that $d^{n-1}\left[\varphi_{0}^{j}, \varphi_{j}^{i}\right]\left(\widetilde{Y}_{i}\right)=\widetilde{Y}_{j}, d^{n-1}\left[\varphi_{0}^{j}, \varphi_{j}^{i}\right]\left(\widetilde{A}_{i}\right)=\widetilde{A}_{j}, d^{n-1}\left[\varphi_{0}^{j}, \varphi_{j}^{i}\right]\left(\widetilde{B}_{i}\right)=\widetilde{B}_{j}$, and $d^{n-1}\left[\varphi_{0}^{j}, \varphi_{j}^{i}\right]\left(\widetilde{C}_{i}\right)=\widetilde{C}_{j}$. Using Proposition $5 n-1$ times we get that the system $\left\{j, \varphi_{j}^{i}\right\}_{j=0}^{\infty}$ contains a short triod $\left\{Y_{j}, A_{j}, B_{j}, C_{j}\right\}_{j=0}^{\infty}$. There is an integer $m$ such that none of the edges $A_{m}, B_{m}$, and $C_{m}$ is contained in the union of the remaining two and $Y_{m}$. Let $T, Y, A, B$, and $C$ be the inverse limits of $\left\{T_{j}, \varphi_{j}^{i}\right\}_{j=0}^{\infty},\left\{Y_{j}, \varphi_{j}^{i} \mid Y_{i}\right\}_{j=0}^{\infty},\left\{A_{j}, \varphi_{j}^{i} \mid A_{i}\right\}_{j=0}^{\infty},\left\{B_{j}, \varphi_{j}^{i} \mid B_{i}\right\}_{j=0}^{\infty}$, and $\left\{C_{j}, \varphi_{j}^{i} \mid C_{i}\right\}_{j=0}^{\infty}$, respectively. Observe that $A \cup Y, B \cup Y$, and $C \cup Y$ are subcontinua of $T$. Since the projection of these continua to $T_{m}$ forms a triod $A_{m} \cup Y_{m}, B_{m} \cup Y_{m}$, and $C_{m} \cup Y_{m}$, the continua $A \cup Y, B \cup Y$, and $C \cup Y$ form a triod in the inverse limits of $\left\{T_{j}, \varphi_{j}^{i}\right\}_{j=0}^{\infty}$. This contradiction completes the proof of the theorem.

Proposition 6. Let $n$ be a positive integer and let $\varphi: T \rightarrow G$ be a simplicial map such that $T$ and $D^{n}(\varphi, T)$ are trees. Suppose that $\psi$ is a simplicial map of a connected graph $C$ onto $T$. Then $d^{n}[\varphi, T]$ are trees. Suppose that $\psi$ is a simplicial map of a connected graph $C$ onto $T$. Then $d^{n}[\varphi, \psi]$ maps $D^{n}(\varphi \circ \psi, C)$ onto $D^{n}(\varphi, T)$. 
Proof. It follows from Propositions 2.15 and 2.16 in [7] that $D(\varphi, T)$, $D^{2}(\varphi, T), \ldots, D^{n-1}(\varphi, T)$ are trees. Since $C$ is connected and every vertex of the tree $T$ is contained in $\psi(C)$, every edge of $T$ is contained in $\psi(C)$. Hence each vertex of $D(\varphi, T)$ is contained in $d[\varphi, \psi](D(\varphi \circ \psi, C))$. Since $D^{j}(\varphi \circ \psi, C)$ is connected for each positive integer $j$, the proposition follows by induction.

Theorem 2. Let $\left\{T_{j}, \varphi_{j}^{i}\right\}_{j=0}^{\infty}$ be an inverse system of trees with simplicial bonding maps $\varphi_{j}^{i}: T_{i} \rightarrow T_{j}$. If the inverse limit (in the topological sense) of $\left\{T_{j}, \varphi_{j}^{i}\right\}_{j=0}^{\infty}$ is atriodic and weakly chainable, then it is chainable.

Proof. To prove that the inverse limit of $\left\{T_{j}, \varphi_{j}^{i}\right\}_{j=0}^{\infty}$ is chainable, it is enough to show that for each integer $m \geq 0$ there is an integer $k>m$ such that $\varphi_{m}^{k}$ can be factored through an arc (see [7, Theorem 3.3]). Since the inverse limit of $\left\{T_{j}, \varphi_{j}^{i}\right\}_{j=0}^{\infty}$ is homeomorphic to the inverse limit of $\left\{T_{j}, \varphi_{j}^{i}\right\}_{j=m}^{\infty}$, it is sufficient to prove that there is a positive integer $k$ such that $\varphi_{0}^{k}$ can be factored through an arc.

Without loss of generality we may assume that the bonding maps are surjective. By Proposition 1, there is an inverse system $\left\{A_{j}, \alpha_{j}^{i}\right\}_{j=0}^{\infty}$ of simplicial arcs with simplicial bonding maps and there is a sequence of simplicial and surjective maps $\sigma_{j}: A_{j} \rightarrow T_{j}, j=0,1, \ldots$, such that $\sigma_{j} \circ \alpha_{j}^{i}=\varphi_{j}^{i} \circ \sigma_{i}$ for every $i \geq j$. By [7, 2.7], there is an integer $n$ such that $D^{n}\left(\sigma_{0}, A_{0}\right)$ is empty. By Theorem 1, there is a positive integer $k$ such that $D^{n}\left(\varphi_{0}^{k}, T_{k}\right)$ is a tree. By Proposition 6,

$$
d^{n}\left[\varphi_{0}^{k}, \alpha_{k}\right]\left(D^{n}\left(\varphi_{0}^{k} \circ \sigma_{k}, A_{k}\right)\right)=D^{n}\left(\varphi_{0}^{k}, T_{k}\right) .
$$

Since $d^{n}\left[\sigma_{0}\right]$ is the empty map and $d^{n}\left[\sigma_{0}\right] \circ d^{n}\left[\sigma_{0}, \alpha_{0}^{k}\right]=d^{n}\left[\varphi_{0}^{k}\right] \circ d^{n}\left[\varphi_{0}^{k}, \alpha_{k}\right]$, the map $d^{n}\left[\varphi_{0}^{k}\right]$ is empty. It follows from [7, Theorem 2.13] that $\varphi_{0}^{k}$ can be factored through an arc.

\section{REFERENCES}

1. H. Cook, W. T. Ingram, and A. Lelek, Eleven annotated problems about continua, preprint.

2. L. Fearnley, Characterizations of continuous images of pseudoarc, Trans. Amer. Math. Soc. 111 (1964), 380-399.

3. A. Lelek, On weakly chainable continua, Fund. Math. 51 (1962), 271-282.

4. __ Disjoint mappings and the span of spaces, Fund. Math. 55 (1964), 199-214.

5. _ On the surjective span and semispan of connected metric spaces, Colloq. Math. 37 (1977), 35-45.

6. W. Lewis, Continuum theory problems, Topology Proc. 8 (1983), 361-394.

7. P. Minc, On simplicial maps and chainable continua, preprint.

8. L. Mohler and L. G. Oversteegen, Reduction and irreducibility for words and tree-words, Fund. Math. 126 (1986), 107-121.

9. L. G. Oversteegen, On reductions of tree-words to (chain-) words, Houston J. Math. 15 (1989), 121-135.

10. __ On span zero and chainability of continua, Houston J. Math. 15 (1989), 573-593.

11. L. G. Oversteegen and E. D. Tymchatyn, Plane strips and the span of continua. I, Houston J. Math. 8 (1982), 129-142.

12. _ Plane strips and the span of continua. II, Houston J. Math. 10 (1984), 255-266. 
13. __ On span of weakly-chainable continua, Fund. Math. 119 (1983), 151-155.

14. __ On span and weakly chainable continua, Fund. Math. 122 (1984), 159-174.

15. _ On span and chainable continua, Fund. Math. 123 (1984), 137-149.

16. University of Houston Problem Book, mimeographed notes.

Department of Foundations, Analysis \& Topology, Auburn University, Auburn UniVERSITY, ALABAMA 36879

E-mail address: PMINC@DUCVAX.AUBURN.EDU 